\title{
Self-Assembly of Protamine Biomacromolecule on Halloysite Nanotubes for Immobilization of Superoxide Dismutase Enzyme
} Bojana Katana, ${ }^{\dagger}$ Paul Rouster, ${ }^{\ddagger}$ Gábor Varga, ${ }^{\S}$ Szabolcs Muráth, ${ }^{\dagger}, \|$ Karine Glinel, ${ }^{\ddagger}$ Alain M. Jonas, ${ }^{\ddagger}$ and Istvan Szilagyi*, ${ }^{\dagger}, \| \odot$

\author{
${ }^{\dagger}$ MTA-SZTE Lendület Biocolloids Research Group, University of Szeged, H-6720 Szeged, Hungary \\ ${ }^{\ddagger}$ Institute of Condensed Matter and Nanosciences - Bio and Soft Matter, Université catholique de Louvain, B-1348 \\ Louvain-la-Neuve, Belgium \\ ${ }^{\S}$ Department of Organic Chemistry, University of Szeged, H-6720 Szeged, Hungary \\ "Interdisciplinary Excellence Center, Department of Physical Chemistry and Materials Science, University of Szeged, H-6720 \\ Szeged, Hungary
}

\section{Supporting Information}

ABSTRACT: An antioxidant material composed of halloysite nanotubes (HNTs), protamine sulfate polyelectrolyte (PSP), and superoxide dismutase (SOD) enzyme was prepared by selfassembly of the PSP and SOD biomacromolecules on the nanoparticulate support. The structural, colloidal and biocatalytic features were assessed. Adsorption of PSP on the oppositely charged HNT surface at appropriate loadings gave rise to charge neutralization and overcharging, which resulted in unstable and stable dispersions, respectively. The formation of a saturated PSP layer on the HNT led to the development of positive surface charge and to remarkable resistance against salt-induced aggregation making the obtained HNT-PSP hybrid suitable for
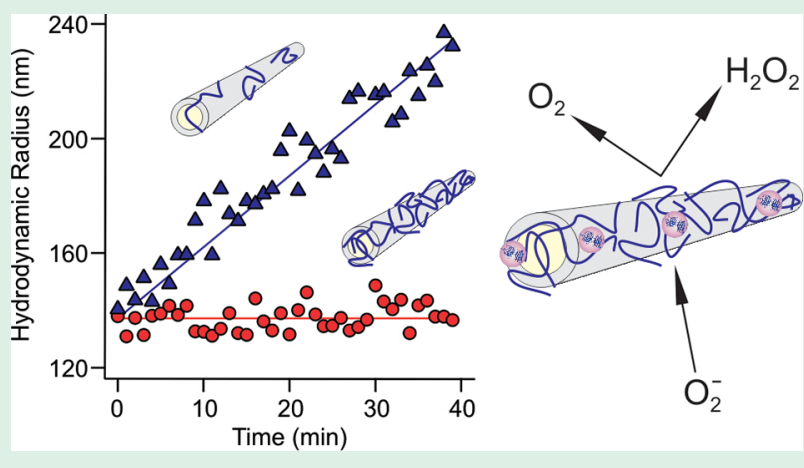
immobilization of negatively charged SOD. No enzyme leakage was observed from the HNT-PSP-SOD composite indicating sufficient structural stability of this material due to electrostatic, hydrophobic, and hydrogen bonding interactions taking place between the particles and the biomacromolecules. Enzymatic assays revealed that SOD kept its functional integrity upon immobilization and showed high activity in superoxide radical dismutation. In this way, stable antioxidant bionanocomposite dispersions were obtained, which can be used as antioxidants in heterogeneous samples.

KEYWORDS: halloysite nanotubes, superoxide dismutase, protamine sulfate, colloidal stability, self-assembly

\section{INTRODUCTION}

Antioxidant enzymes are the most efficient defense systems against reactive oxygen species (ROS), which are responsible for the development of several diseases including cancer due to their high reactivity against lipids and other cell constituent biomolecules. ${ }^{1}$ On the other hand, their harmful effect leads to the damage of commercial products during chemical manufacturing processes in the food ${ }^{2}$ and cosmetic ${ }^{3}$ industries. Among them, superoxide radical anions are the most adverse ones, since they serve also as precursors to other ROS such as peroxides. ${ }^{4}$ The natural defense systems developed during evolution against superoxide radicals are the so-called superoxide dismutase (SOD) enzymes. ${ }^{5}$ They are able to effectively decompose superoxide radicals to molecular oxygen and hydrogen peroxide. The latter one is subsequently converted to oxygen and water by catalase and peroxidase enzymes in the cellular environment. ${ }^{6}$

Despite its great promise in biomedical and industrial applications as an antioxidant agent, ${ }^{7}$ the supplementation of
SOD is complicated due to its high sensitivity to environmental conditions including $\mathrm{pH}$, temperature, and the presence of enzyme inhibitor molecules. To overcome this problem, immobilization of SOD attracted considerable attention in the scientific community over the past decade. ${ }^{8-11}$ The role of the solid support is (i) to defend the enzyme during the supplementation process and (ii) to deliver SOD to the desired location in the living organisms or in the industrial samples. For example, SOD enzyme was embedded in mesoporous silica and conjugation with a cell-penetrating protein led to excellent activity in reducing cellular oxidative stress. $^{12}$ In another approach, SOD was captured in polyacrylamide hydrogel nanoparticles and remained active upon immobilization. ${ }^{13}$ Due to the tuned permeability of the carrier, the substrates and the products freely diffused in the

Received: October 16, 2019

Accepted: December 3, 2019

Published: December 3, 2019 
system, while the enzyme was protected against denaturation by inhibitors. To avoid enzyme leakage, SOD was covalently attached to carbon nanoparticles and the obtained material maintained long-term superoxide radical scavenging activity and was found to be nontoxic. ${ }^{14}$ The selection of the carrier nanomaterials is especially important, since denaturation of the enzyme may occur due to changes in its conformation upon adsorption on the surface of the solid support. Therefore, polyelectrolyte functionalization of the carrier clay ${ }^{9}$ or titania ${ }^{10}$ particles were performed to avoid enzyme degradation and to improve the colloid stability of the system. The latter issue is crucial, given the fact that the applications of the enzymenanoparticle conjugates are foreseen mostly in liquid medium, that is, in biofluids and in industrial liqueurs, where aggregation of the biocatalytic particles gives rise to loss of enzymatic activity.

Due to their advantageous properties such as biocompatibility, high aspect ratio, and availability in large scale, ${ }^{15-17}$ halloysite nanotubes (HNTs) are promising candidates as solid supports in immobilization processes. ${ }^{18,19}$ Moreover, their advantages over other clay particles include the relatively narrow size distribution and possible activation of the surface functional groups. ${ }^{20}$ These features facilitate the formation of fine HNT dispersions in aqueous medium. Enzyme attachment on the outer surface or in the lumen, which are oppositely charged at physiological $\mathrm{pH}$, attracted considerable contemporary interest in the scientific community. Accordingly, laccase, ${ }^{21}$ lipase $^{22}$ amylase, ${ }^{23}$ lactamase, ${ }^{20}$ urease, $^{24}$ and glucose oxidase $^{25}$ were immobilized in/on HNTs or their surface functionalized derivatives. The general conclusion was that HNT is a promising candidate for enzyme delivery processes; however, the substrate diffusion into the lumen and product release from it are often the limits of the HNT application as a carrier in enzyme immobilization. The HNTs have already proven to act as sufficient delivery agents for molecular antioxidants such as quercetin, ${ }^{26}$ curcumin, ${ }^{27}$ and resveratrol, ${ }^{28}$ nevertheless, immobilization of antioxidant enzymes was not reported so far.

Besides, in many of the bioapplications of HNTs, surface functionalization with macromolecules is necessary to strengthen the adsorption of the guest molecules and to avoid unwanted aggregation processes. The interaction between HNTs and polyelectrolytes (heparin, ${ }^{29}$ poly(ethylene imine $),{ }^{20}$ poly (acrylamide-co-diallyldimethylammonium chloride), ${ }^{30}$ poly(amido amine) dendrimers, ${ }^{31}$ and chitosan ${ }^{32}$ ) was studied to some extent. Furthermore, immobilization of drug molecules among polyelectrolyte layers adsorbed on the surface of HNT was also carried out. ${ }^{33}$ The general remarks were that polyelectrolyte adsorption onto HNTs significantly influences the surface features and that the adsorption process plays a major role in the aggregation mechanism of the nanotubes. Despite these works reported earlier, the fundamental understanding of the relation between the surface charge properties and the colloidal stability of polyelectrolyte decorated HNTs is missing.

The present study, therefore, aims at the development of stable colloids of a nanocomposite consisting of self-assembled biomacromolecules such as protamine sulfate polyelectrolyte (PSP) and SOD enzyme on the HNT, which served as solid support. The influence of the experimental conditions such as biomacromolecule dose, $\mathrm{pH}$, and ionic strength on the colloidal and structural stability of HNT-PSP-SOD was explored by spectroscopy, microscopy, and light scattering techniques, whereas the superoxide radical scavenging activity was probed in biochemical assays.

\section{EXPERIMENTAL SECTION}

Materials. Biomacromolecules including PSP (from salmon sperm, molecular weight of 5000-10000 g/mol), xanthine oxidase (from bovine milk, lyophilized powder, $0.4-1.0$ units $/ \mathrm{mg}$ protein), and SOD (from bovine liver, lyophilized powder, $\geq 1500$ units/mg protein) were purchased from Sigma-Aldrich. Nitro blue tetrazolium (NBT), $\mathrm{KCl}, \mathrm{KBr}$, ethanol, and xanthine were bought from VWR. Coomassie Brilliant Blue (G-250) was purchased from Fisher Scientific. Ortho-phosphoric acid $\left(\mathrm{H}_{3} \mathrm{PO}_{4}, 85 \%\right)$ was acquired from Carl Roth. All chemicals were used as received. The measurements were carried out at $25{ }^{\circ} \mathrm{C}$ and $\mathrm{pH} 7$. High purity water from a VWR Purity TU+ device was used for all the sample preparations. Salt solutions and water applied for light scattering experiments were filtered through a $0.1 \mu \mathrm{m}$ syringe filter (Millex).

Alkali Activation of HNTs. The raw HNT powder was purchased from Sigma-Aldrich. To improve the surface properties, alkali activation was performed using a method reported earlier. ${ }^{20}$ Briefly, the commercial (raw) HNT powder was dispersed at a concentration of $20 \mathrm{~g} / \mathrm{L}$ in a $14.5 \mathrm{mM} \mathrm{KOH}$ solution under magnetic stirring for 24 h. The dispersion was centrifuged at $10000 \mathrm{rpm}$ for $10 \mathrm{~min}$. The supernatant was removed and the slurry was redispersed in water. The centrifugation steps were repeated until the $\mathrm{pH}$ of the supernatant was around 7. Afterwards, the slurrie was recovered and put in a glass container in an oven at $110^{\circ} \mathrm{C}$ for $15 \mathrm{~h}$ to obtain a dry powder. The obtained hydroxylated HNTs were then dispersed in water at a concentration of $10000 \mathrm{mg} / \mathrm{L}$ to be used as a stock dispersion for sample preparation. In the following, the term HNT will refer to the hydroxylated HNT unless mentioned otherwise. To compare the dispersion characteristics of the raw and activated HNT, the electrophoretic mobilities, hydrodynamic radii and polydispersity indices were determined in stable dispersions by light scattering. The mobilities were the same $\left(-2.5 \times 10^{-8} \mathrm{~m}^{2} /(\mathrm{V} \mathrm{s})\right)$ for both samples within the experimental error, the radii were 146 and 225 $\mathrm{nm}$, while the polydispersities were 20 and $25 \%$ for the activated and the raw HNT, respectively. These data clearly indicate the improvement of the particle size distribution after the alkaline treatment.

Electrophoretic Mobility. The electrophoretic light scattering measurements were performed with a Litesizer 500 instrument (Anton Paar). In a sample preparation process, $0.2 \mathrm{~mL}$ of stable HNT dispersion of $100 \mathrm{mg} / \mathrm{L}$ concentration was added to a $1.8 \mathrm{~mL}$ solution composed of PSP and/or $\mathrm{KCl}$ at appropriate concentrations. The samples were allowed to rest for $2 \mathrm{~h}$ at room temperature before measuring the electrophoretic mobility. An equilibration time of 1 min was also applied in the device prior the measurements that were performed in $350 \mu \mathrm{L}$ omega-shaped plastic cuvettes (Anton Paar). The average value of five individual measurements was reported.

The electrophoretic mobility of the bar HNT was found to be negative in a wide range of $\mathrm{pH}$ due to the abundance of the deprotonated silanol groups on the outer surface (SI Figure S1). Electrophoresis can only detect the net charge of the particles; therefore, the contribution of the positively charged protonated Al$\mathrm{O}-\mathrm{H}$ groups ${ }^{34}$ of the inner surface of the HNT could not be assessed by this technique. A steep decrease in the values was observed in the $\mathrm{pH} 4-6$ regime, since the $\mathrm{p} K_{\mathrm{a}}$ of the silanol groups falls in this range and the mobility was almost constant above $\mathrm{pH} 7$.

Dynamic Light Scattering. To assess the possible aggregation processes in the samples, time-resolved dynamic light scattering (DLS) measurements were carried out with a Litesizer 500 instrument (Anton Paar) equipped with a $40 \mathrm{~mW}$ semiconductor laser (658 nm wavelength) operating in the backscattering mode at a scattering angle of $175^{\circ}$. To determine the hydrodynamic radii, the correlation functions were collected for $20 \mathrm{~s}$, and the decay rate constants were calculated using the cumulant analysis. The diffusion coefficients were converted to hydrodynamic radius with the StokesEinstein equation. ${ }^{35}$ The particle concentration was kept constant (10 

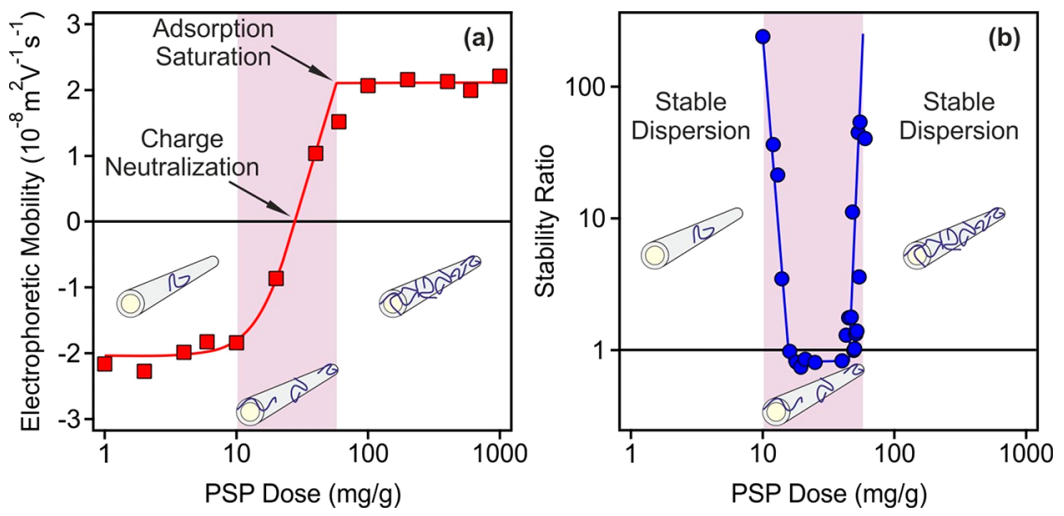

Figure 1. (a) Variation of the electrophoretic mobility and (b) stability ratio of HNT as a function of the PSP dose. The measurements were performed at $\mathrm{pH} \mathrm{7,1} \mathrm{mM} \mathrm{ionic} \mathrm{strength} \mathrm{adjusted} \mathrm{by} \mathrm{KCl}$ and $10 \mathrm{mg} / \mathrm{L} \mathrm{HNT}$ concentration. The $\mathrm{mg} / \mathrm{g}$ unit on the $x$-axis refers to mg of PSP per one gram of HNT. The PSP dose range corresponding to the aggregation regime is shaded. The lines serve to guide the eyes.

$\mathrm{mg} / \mathrm{L}$ ), and the PSP and/or $\mathrm{KCl}$ concentration was varied. Otherwise, the sample preparation procedure was the same as in the electrophoretic studies with the exception that the DLS measurements were acquired immediately after adding the HNT stock dispersion and vortexing the sample for $25 \mathrm{~s}$. The samples were equilibrated for $30 \mathrm{~s}$ in the instrument before data collection was started. The colloidal stability was expressed in terms of stability ratio. ${ }^{9,10,36-38}$ Its value is unity in the case of unstable dispersions, where the particle aggregation is controlled solely by their diffusion, whereas higher stability ratios refer to slower aggregation and thus, to more stable samples.

IR Spectroscopy. FT-IR spectroscopy was applied to determine the presence of the PSP and SOD in the composites. A BIO-RAD Digilab Division FTS-65A/896 device was used to record the spectra, for which 256 scans were performed with $4 \mathrm{~cm}^{-1}$ resolution in the $4000-600 \mathrm{~cm}^{-1}$ wavenumber range. The spectrum of each sample was taken in the diffuse reflectance mode (DRS) fixing the incident angle in the $45^{\circ}$ position. To prepare the samples, $30 \mathrm{mg}$ of the solid material measured with analytical accuracy was mixed with $300 \mathrm{mg}$ of $\mathrm{KBr}$.

Electron Microscopy. Morphological studies were carried out using scanning electron microscopy (SEM, Hitachi S-4700) and transmission electron microscopy (TEM, FEI TECNAI G220 XTWIN). For SEM, $10 \mathrm{kV}$ accelerating voltage was applied on the nanotubes mounted on a double sided carbon tape. Before measurements, the samples were coated with gold via physical vapor deposition using a Quorum Technologies SC7620 device. For TEM measurements, the dispersions of the particles were dried on copper-carbon mesh grid, and $200 \mathrm{kV}$ accelerating voltage was used for imaging in the bright field mode.

Thermal Gravimetric Analysis. To test the thermal decomposition of the bare and functionalized HNTs, thermal gravimetric analysis (TGA) was carried out on a Mettler Toledo TGA/ SDTA851e instrument under nitrogen atmosphere. The samples were allowed to equilibrate for $5 \mathrm{~min}$ at $30^{\circ} \mathrm{C}$ in the instrument before being submitted to a heating rate of $10{ }^{\circ} \mathrm{C} / \mathrm{min}$ up to $700{ }^{\circ} \mathrm{C}$. For the TGA experiments, solid samples were obtained by centrifugation of the dispersions and subsequent drying in a desiccator at room temperature.

Bradford Test. The amount of SOD adsorbed on the PSPmodified HNT (HNT-PSP) was quantified by the Bradford test. ${ }^{39}$ The Bradford reagent was prepared in a $250 \mathrm{~mL}$ volumetric flask by dissolving $25 \mathrm{mg}$ of Coomassie Brilliant Blue in $12.5 \mathrm{~mL}$ of ethanol followed by the addition of $25 \mathrm{~mL}$ of phosphoric acid. Then, the solution was completed to $250 \mathrm{~mL}$ with ultrapure water. A calibration curve was obtained by using standard SOD solutions with concentrations in the range from 1 to $100 \mathrm{mg} / \mathrm{L}$. These solutions were prepared by mixing $400 \mu \mathrm{L}$ of a SOD solution at a given concentration with $1.6 \mathrm{~mL}$ of the Bradford reagent. The mixture was then allowed to equilibrate during $5 \mathrm{~min}$ prior to the acquisition of the
UV-vis spectrum, which was recorded with a Genesys 10S spectrophotometer (Thermo Scientific). The absorbance bands centered at 465 and $595 \mathrm{~nm}$ corresponding to the free dye and to both the SOD-dye complex and free dye, respectively, were monitored. To quantify the amount of SOD adsorbed on the HNT-PSP particles, dispersions were prepared by mixing $10 \mathrm{mg}$ of SOD per gram of HNT-PSP to obtain a final SOD concentration of $50 \mathrm{mg} / \mathrm{L}$. Then, these dispersions were centrifuged and the supernatant was recovered and filtered through a syringe filter of $0.1 \mu \mathrm{m}$ pore size to remove any HNT-PSP-SOD that may still be present in order to only assess the amount of free SOD that did not adsorb on the HNT-PSP particles. Finally, $400 \mu \mathrm{L}$ of this supernatant was mixed with $1.6 \mathrm{~mL}$ of the Bradford reagent. The difference between the absorbance values recorded at 595 and $465 \mathrm{~nm}$ wavelengths were used to obtain the amount of free SOD present after removing the HNT-PSP-SOD from the system.

SOD Activity. To determine the SOD activity of the developed material (HNT-PSP-SOD), the Fridovich method ${ }^{40}$ was applied. However, the enzymatic assay protocol had to be slightly modified to adapt to the light scattering events resulting from the dispersed particles. Each sample was composed of xanthine $(3 \mathrm{mM}, 0.2 \mathrm{~mL})$, NBT $(3 \mathrm{mM}, 0.1 \mathrm{~mL})$, xanthine oxidase $(3 \mathrm{mg} / \mathrm{mL}, 0.3 \mathrm{~mL})$, and the stock solution containing the catalyst (free or immobilized SOD with various concentrations) in phosphate buffer to reach a final volume of $3 \mathrm{~mL}$. The phosphate buffer concentration in the samples was $1 \mathrm{mM}$ and the $\mathrm{pH}$ was set to 7 . Once all the reagents were mixed together, the samples were left to equilibrate for $1 \mathrm{~min}$ prior to monitoring the increase of the absorbance band at $565 \mathrm{~nm}$ for $5 \mathrm{~min}$. Such an increase of the absorbance, caused by the increasing concentration of the blue colored product resulting from the NBT-superoxide radical reaction, was determined for several SOD concentrations in the sample. Furthermore, due to the scattering of the HNT particles, the corresponding HNT-PSP samples devoid of SOD were used as references. The inhibition of the NBT-superoxide radical reaction was then calculated and the activity in dismutation of the radicals by the enzyme was expressed in $\mathrm{IC}_{50}$ values, which correspond to the SOD concentration needed to decompose $50 \%$ of the radicals formed in the probe reaction. The average error of the method is about $5 \%$.

\section{RESULTS AND DISCUSSION}

Self-Assembly of PSP on HNT. As a natural polyelectrolyte, PSP was used to functionalize the surface of HNT. The aim of such a functionalization was (i) to obtain biocompatible nanotubes for further enzyme immobilization, (ii) to enhance the strength of attractive enzyme-particle interactions, and (iii) to tune the colloidal stability of the HNT. The PSP possesses positive charge at physiological $\mathrm{pH}$ due to the high occurrence of the arginine amino acids of positively charged side chain groups. ${ }^{41}$ PSP has proven to strongly adsorb on oppositely 

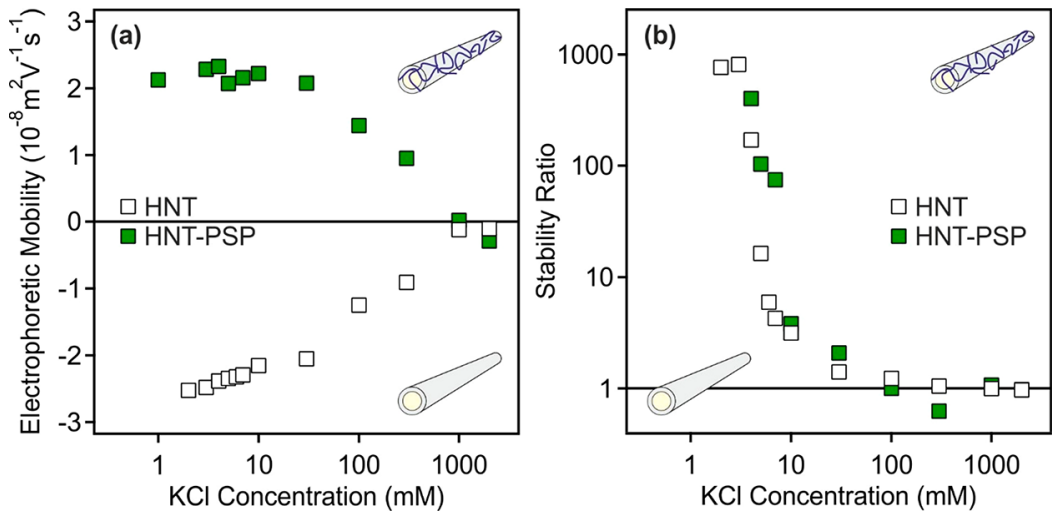

Figure 2. (a) Electrophoretic mobility and (b) stability ratio of HNT (empty symbols) and HNT-PSP (filled symbols) versus KCl concentration. The measurements were performed at neutral $\mathrm{pH}$ and $10 \mathrm{mg} / \mathrm{L}$ particle concentration. The HNT-PSP particles were prepared with a PSP dose of $100 \mathrm{mg} / \mathrm{g}$.

charged particles leading to their overcharging and thus, to their charge inversion. ${ }^{38}$

The electrophoretic mobility was first measured at different PSP doses, where the particle concentration, ionic strength, and $\mathrm{pH}$ were kept constant. As shown in Figure 1a, negative electrophoretic mobilities of about $-2.0 \times 10^{-8} \mathrm{~m}^{2} /(\mathrm{V} \mathrm{s})$ were recorded at low PSP loadings. This value is similar to the mobility of the bare HNT at the selected ionic strength (1 $\mathrm{mM}$ ). For PSP doses higher than $10 \mathrm{mg} / \mathrm{g}$, a sharp increase of the electrophoretic mobility was observed, which indicates the adsorption of PSP chains onto the oppositely charged surface of HNT.

Such an adsorption led to charge neutralization and overcharging at appropriate polyelectrolyte doses. At PSP concentrations higher than $100 \mathrm{mg} / \mathrm{g}$, the mobility remained constant around $2.0 \times 10^{-8} \mathrm{~m}^{2} /(\mathrm{V} \mathrm{s})$ due to the formation of a saturated PSP layer on the HNT surface. Further added polyelectrolyte did not adsorb and remained dissolved in the bulk solution. The magnitude of the mobilities of the bare and PSP-coated HNT is the same within the experimental error, indicating very similar magnitude of the surface charge in both cases. Similar results were reported with other inorganic particles in the presence of oppositely charged polyelectrolytes. $^{42-46}$

Aggregation of the PSP-modified HNT was investigated at different PSP doses by time-resolved DLS (SI Figure S2). The hydrodynamic radius was constant within the experimental error at a PSP dose of $10 \mathrm{mg} / \mathrm{g}$, and a slight increase was detected at a dose of $50 \mathrm{mg} / \mathrm{g}$; however, a significant increase of the hydrodynamic size of the particles was observed at a dose of $20 \mathrm{mg} / \mathrm{g}$. Comparing with the electrophoretic mobility measurements discussed above, these three doses are below, above, and at the charge neutralization point, respectively. The trend in the stability ratio followed a U-shape curve with a minimum reached for the PSP dose corresponding to the charge neutralization point (Figure $1 \mathrm{~b}$ ).

The aggregation was rapid, i.e., the stability ratio was close to one, at the minimum, while the dispersions were stable before and after this regime, where the particles possessed sufficiently high surface charge. These results can be qualitatively described by the DLVO (Derjaguin, Landau, Verwey, and Overbeek) theory. ${ }^{47}$ Accordingly, repulsive electrical double layer forces do not exist at charge neutralization owing to the lack of surface charge, therefore, van der Waals attractions destabilize the dispersions due to particle aggregation. The bottom line of these results is that HNT modified with a PSP layer adsorbed at saturation (HNTPSP, obtained for a PSP dose of $100 \mathrm{mg} / \mathrm{g}$ ) form stable colloidal samples consisting of homogeneously distributed primary particles of high surface area. On the basis of these results and due to the fact that SOD is negatively charged at neutral $\mathrm{pH}$, enzyme adsorption is expected to occur on these HNT-PSP hybrid particles.

Effect of Surface Functionalization on the Resistance against Salt-Induced Aggregation. To further assess the colloidal stability of the HNT-PSP particles (prepared with a PSP dose of $100 \mathrm{mg} / \mathrm{g}$ ), the charging and aggregation processes were investigated in $\mathrm{KCl}$ solutions of different concentrations by electrophoresis and DLS. The effect of the adsorbed PSP layer on the stability of the HNT-PSP dispersions was explored by comparing the obtained data with the ones observed with the bare HNT (Figure 2). The tendencies observed for the variation of the electrophoretic mobility and the stability ratio data with increasing $\mathrm{KCl}$ concentration were similar in both cases. The magnitude of the mobility decreased with the $\mathrm{KCl}$ concentration increasing due to charge screening by the salt constituents and remained close to zero at high $\mathrm{KCl}$ concentration (Figure 2a).

Aggregation of bare and PSP-coated HNT was investigated in the same $\mathrm{KCl}$ concentration range by time-resolved DLS, and it was found that the speed of aggregation increased with $\mathrm{KCl}$ concentration. The hydrodynamic radius was constant within the experimental error at low ionic strength, while it increased with time once the $\mathrm{KCl}$ concentration was higher than $3 \mathrm{mM}$. The slopes of the hydrodynamic radius versus time plots increased with the increase of the ionic strength up to a threshold value of $30 \mathrm{mM}$ (SI Figure S3), where they remained constant above it. Stability ratios were determined at several $\mathrm{KCl}$ concentrations and the slow and fast aggregation regimes were defined (Figure $2 b$ ).

In general, the stability ratios decreased until $30 \mathrm{mM}$ in both systems and remained constant for higher $\mathrm{KCl}$ concentrations. Therefore, this value is the so-called critical coagulation concentration (CCC). Such a sharp transition between the fast and slow aggregation regime indicates the presence of DLVOtype forces reported earlier for charged colloidal particles dispersed in electrolyte solutions. ${ }^{9,10,37,38,44}$ The CCC value of the HNT-PSP was very similar to the one determined for the HNT. Due to the fact that similar surface charge was assumed for the bare and coated HNT from the electrophoretic 
mobility measurements, the identical CCC values confirm that the stabilizing forces originate from the overlap of the electrical double layers, as predicted by the DLVO model.

Despite the opposite sign of charge of the particles, one can conclude that the dispersion characteristics of HNT and HNTPSP are very similar, and both nanotubes form stable dispersions below $30 \mathrm{mM} \mathrm{KCl}$. However, HNT-PSP was selected for the SOD immobilization process as stronger enzyme adsorption is expected on their positively charged surface than in the case of negatively charged bare HNT.

Immobilization of SOD. Based on our previous experiences with enzyme immobilization on polyelectrolytefunctionalized particles, ${ }^{9,10}$ the HNT-PSP-SOD bionanocomposite was prepared by adsorbing the SOD on the HNT-PSP prepared with a SOD dose of $10 \mathrm{mg} / \mathrm{g}$. Accordingly, the obtained HNT-PSP-SOD nanotubes contained $100 \mathrm{mg}$ of PSP and $10 \mathrm{mg}$ of SOD together with $1 \mathrm{~g}$ of HNT. The isoelectric point of the enzyme is at $\mathrm{pH} 4.95,{ }^{48}$ therefore it is negatively charged at neutral $\mathrm{pH}$, the condition, under which the experiments were carried out. It is expected that the enzyme strongly adsorbs on the oppositely charged material. However, further confirmation of the successful immobilization was necessary.

Therefore, the adsorption process was quantified by using the Bradford method ${ }^{39}$ to determine the SOD concentration in the solution after removing the HNT-PSP-SOD particles by centrifugation. In other words, if SOD partitioning takes place between the solution phase and the particle surface, its presence should be detected by the Bradford test after removing the HNT-PSP-SOD from the sample. Applying such a test, potential enzyme leakage from the composite can be probed. The results, together with four reference measurements performed on SOD solutions of different concentrations, are shown in SI Figure S4. The absorbance decreases at $465 \mathrm{~nm}$ and increases at $595 \mathrm{~nm}$ when increasing the enzyme concentration. These variations of the intensity of absorption bands result from the binding of the dye to the SOD. The analysis of the spectra revealed that the spectrum measured for the supernatant obtained after SOD adsorption on HNT-PSP was similar to the one of the reference sample devoid of SOD molecules. This finding clearly shows that the enzyme quantitatively adsorbed on the HNT-PSP, and that no partitioning between the bulk and surface occurred, when an enzyme loading of $10 \mathrm{mg} / \mathrm{g}$ is applied. The fact that SOD was not detected after removing the HNT-PSP-SOD hybrid from the dispersions indicates negligible enzyme leakage from the composite. It was assumed that the strong attractive forces between the SOD molecules and the solid support were achieved through electrostatic and hydrophobic interactions as well as by hydrogen bonding.

To further prove the adsorption of the enzyme on the HNTPSP, the FT-IR spectrum of the HNT-PSP-SOD composite was measured and compared to the spectra of the bare HNT and HNT-PSP (Figure 3).

The FT-IR spectrum of HNT exhibits two different $\mathrm{Al}-\mathrm{OH}$ stretching bands at about 3702 and $3615 \mathrm{~cm}^{-1}$. ${ }^{49}$ The intense peak at $1089 \mathrm{~cm}^{-1}$ is associated with the stretching vibration mode of apical $\mathrm{Si}-\mathrm{O}$, while the absorption band at $1021 \mathrm{~cm}^{-1}$ is assigned to the stretching vibration of $\mathrm{Si}-\mathrm{O}-\mathrm{Si}^{50}$ In addition, the intense peak around $900 \mathrm{~cm}^{-1}$ can be identified as a single $\mathrm{Al}-\mathrm{OH}$ bending vibration. ${ }^{51}$ Moreover, in the FTIR spectra of the HNT-PSP and HNT-PSP-SOD composite, the absorption bands at approximately 1677 and $1541 \mathrm{~cm}^{-1}$,

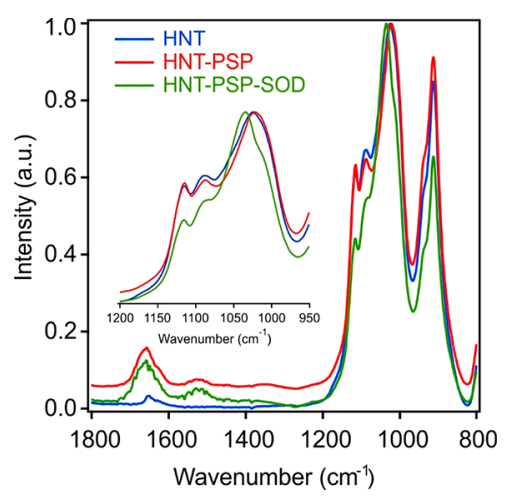

Figure 3. Diffuse reflectance FT-IR spectra of bare HNT, HNT-PSP, and HNT-PSP-SOD in solid state.

which are related to the shifted amide I and amide II vibrations, are attributed to the presence of PSP. ${ }^{52}$ In the case of the HNT-PSP-SOD hybrid, the peak at $1035 \mathrm{~cm}^{-1}$ is assigned to the shifted $\mathrm{C}-\mathrm{O}$ stretching vibration of the histidine unit of the SOD enzyme. ${ }^{53}$ The observed shifts of the characteristic bands indicate the strong interaction between HNT and PSP and SOD.

The possible changes in the nanoparticle morphology after surface modification were assessed by TEM and SEM. Due to the fact that the images were recorded after drying the sample, nanotube aggregates were observed. However, the typical morphological features of the bare and modified nanotubes can be identified. The tubular morphology of both HNT and HNT-PSP-SOD particles is clearly visible on the SEM micrographs (SI Figure S5). Furthermore, TEM images revealed the intrinsic layered-tubular structural motifs of the nanotubes (Figure 4). The length of the particles typically fell between 200 and $500 \mathrm{~nm}$ with ca. $50-60 \mathrm{~nm}$ of outer and 10 $\mathrm{nm}$ inner diameter, and the samples were moderately polydisperse in the lateral dimension. These features were unaltered after coating the tubes with PSP polyelectrolyte and SOD enzyme, thus the surface modification did not change the morphology of the carrier.

In addition, TGA measurements were carried out with the HNT, HNT-PSP, and HNT-PSP-SOD samples to study the thermal decomposition of the materials (SI Figure S6). For all samples, the dehydration occurred between 30 and $200{ }^{\circ} \mathrm{C}$, which is typical for HNT derivatives. ${ }^{54}$ Thereafter, the partial degradation of the organic compounds took place in the range of $200-400{ }^{\circ} \mathrm{C}$. The decomposition of the organic content continued between 400 and $700{ }^{\circ} \mathrm{C}$, and this process was accompanied by the dehydroxylation of the $\mathrm{HNT}^{55}$ in this temperature range. The TGA curves were identical within the experimental error for the HNT-PSP and HNT-PSP-SOD due to the low amount $(10 \mathrm{mg} / \mathrm{g})$ of the enzyme present in the latter composite. However, a significant difference was observed between the thermal decomposition of HNT and HNT-PSP evidencing the presence of the PSP macromolecules on the HNT surface.

A remarkable stability of the HNT-PSP composite in aqueous dispersion was concluded in the previous chapter. Moreover, the effect of SOD adsorption on the electrophoretic mobilities and stability ratios was also studied to assess the charging and aggregation processes of the HNT-PSP-SOD containing $10 \mathrm{mg} / \mathrm{g}$ enzyme. Therefore, electrophoretic mobilities and hydrodynamic radii of HNT-PSP were measured in the $0-30 \mathrm{mg} / \mathrm{g}$ SOD concentration range (SI 



Figure 4. TEM micrographs of the bare HNT (left column) and HNT-PSP-SOD (right column) materials. The images were taken after drying 10 $\mathrm{mg} / \mathrm{L}$ dispersions.

Figure S7). The mobilities were the same $\left(2.3 \times 10^{-8} \mathrm{~m}^{2} /(\mathrm{V}\right.$ s)) within the experimental error and they were very similar to the value determined for HNT-PSP $\left(2.0 \times 10^{-8} \mathrm{~m}^{2} /(\mathrm{V} \mathrm{s})\right)$ indicating that enzyme adsorption at such low doses did not affect the surface charge of HNT-PSP. Besides, no particle aggregation was detected under the above experimental conditions, and the hydrodynamic radii were about $182 \mathrm{~nm}$ in the $0-30 \mathrm{mg} / \mathrm{g}$ dose range. In other words, the remarkable stability of the HNT-PSP remained unchanged upon SOD adsorption. Similar observations were published earlier ${ }^{10}$ with composites containing SOD immobilized on particle-polyelectrolyte systems.

Superoxide Radical Scavenging Activity. The native and the immobilized SOD enzymes were tested in the dismutation of superoxide radicals performed in biochemical test reactions. ${ }^{40}$ In these assays, superoxide radicals are produced during oxidation of xanthine to uric acid by xanthine oxidase. The forming radicals reduce NBT to formazan, and this reaction is accompanied by a color change from yellow to blue, which is followed by spectrophotometry. Indeed, when SOD is present in the system, it captures the radicals, that is, inhibits their reaction with NBT. The enzyme activity was expressed in terms of $\mathrm{IC}_{50}$, which is the SOD concentration required for $50 \%$ inhibition or to capture $50 \%$ of the superoxide radicals forming in the assay. The obtained reaction inhibition values as a function of the SOD concentration are presented in Figure 5.

The shape of the inhibition curves was very similar for both SOD and HNT-PSP-SOD indicating that the enzyme kept its

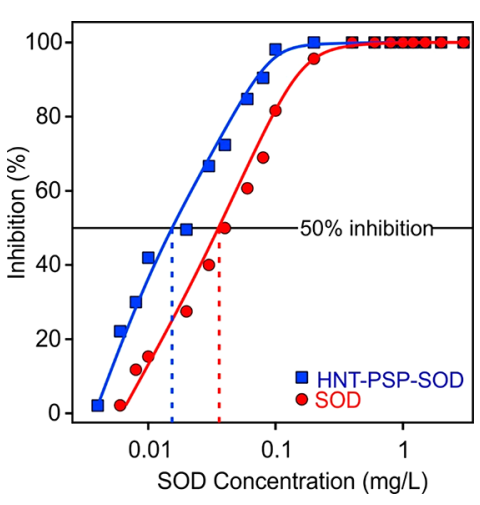

Figure 5. Inhibition of the reaction between NBT and superoxide radicals by SOD (circles) and HNT-PSP-SOD (squares). The solid lines are fits used to calculate the $\mathrm{IC}_{50}$ values. The average measurement error is about $5 \%$. 
functional integrity upon immobilization on the PSP-modified HNT. Complete inhibition (100\%) was reached in both cases. The $\mathrm{IC}_{50}$ value was found to be slightly smaller for HNT-PSPSOD $(0.02 \mathrm{mg} / \mathrm{L})$ than for native SOD $(0.04 \mathrm{mg} / \mathrm{L})$. This increase in the activity most likely originates from the electrostatic interactions between the HNT-PSP-SOD material and the substrate molecules. In other words, the negatively charged superoxide radicals are certainly attracted to the positively charged protamine chains on the surface so that they are available for the immobilized SOD in larger amounts. Considering the fact that enzyme immobilization often leads to significant loss of the enzyme activity, ${ }^{9,56}$ the above results are remarkable.

Thus, the obtained HNT-PSP-SOD hybrid is a promising antioxidant candidate due to its high scavenging activity. In addition, it possesses remarkable colloidal and structural stability, which makes the composite an advantageous biocatalyst in applications. One of its potential utilizations can be in antioxidant therapy for inflammatory bowel diseases. $^{57}$ In such biomedical applications, the formation of a protein corona on the particle surface usually further improves the colloidal stability of the nanocomposites. ${ }^{58,59}$

\section{CONCLUSIONS}

An antioxidant nanocomposite consisting of HNT with selfassembled PSP and SOD biomacromolecules on the outer surface was developed. The PSP showed high affinity to the oppositely charged nanotubes giving rise to significant changes in the surface charge properties by increasing the polyelectrolyte loading. The aggregation of the particles was tuned by varying the PSP dose, and stable dispersions were observed once the amount of the PSP biomacromolecule was high enough to cover the entire outer surface of the HNTs. The SOD adsorption on the PSP-coated nanotubes was confirmed by spectroscopic techniques, and the results indicated not only successful immobilization of SOD, but excellent structural stability of the hybrid system. The immobilization procedure did not affect the nanoscale morphology of the nanotubes. Enzymatic assays revealed that SOD kept its structural and functional integrity upon immobilization, since the HNT-PSPSOD material showed SOD-like activity very similar to the one determined for the support-free enzyme. The slight increase in the dismutation ability was attributed to the advantageous electrostatic interaction between the composite surface and the substrate molecules. These results indicate that the obtained HNT-PSP-SOD dispersion can be considered as a highly active antioxidant with the benefits of the heterogenization process, for example, easier separation from the reaction mixture.

\section{ASSOCIATED CONTENT}

\section{S Supporting Information}

The Supporting Information is available free of charge at https://pubs.acs.org/doi/10.1021/acsabm.9b00953.

$\mathrm{pH}$ and SOD dose-dependent electrophoretic mobilities, SEM images, results of DLS and TGA measurements (PDF)

\section{AUTHOR INFORMATION}

\section{Corresponding Author}

*E-mail: szistvan@chem.u-szeged.hu.

ORCID $\odot$

Karine Glinel: 0000-0002-2000-0169
Alain M. Jonas: 0000-0002-4083-0688

Istvan Szilagyi: 0000-0001-7289-0979

Notes

The authors declare no competing financial interest.

\section{ACKNOWLEDGMENTS}

This research was financially supported by the Lendület program of the Hungarian Academy of Sciences (96130) and by the Ministry of Human Capacities, Hungary (20391-3/ 2018/FEKUSTRAT). K.G. is a Senior Research Associate of the F.R.S.-FNRS. The support from the University of Szeged Open Access Fund (4502) is gratefully acknowledged.

\section{REFERENCES}

(1) Winterbourn, C. C. Reconciling the chemistry and biology of reactive oxygen species. Nat. Chem. Biol. 2008, 4, 278-286.

(2) Finley, J. W.; Kong, A. N.; Hintze, K. J.; Jeffery, E. H.; Ji, L. L.; Lei, X. G. Antioxidants in foods: State of the science important to the food industry. J. Agric. Food Chem. 2011, 59, 6837-6846.

(3) Morsella, M.; d'Alessandro, N.; Lanterna, A. E.; Scaiano, J. C. Improving the sunscreen properties of $\mathrm{TiO}_{2}$ through an understanding of its catalytic properties. ACS Omega 2016, 1, 464-469.

(4) Alfadda, A. A.; Sallam, R. M. Reactive oxygen species in health and disease. J. Biomed. Biotechnol. 2012, 2012, 936486.

(5) Tainer, J. A.; Getzoff, E. D.; Richardson, J. S.; Richardson, D. C. Structure and mechanism of copper, zinc superoxide-dismutase. Nature 1983, 306, 284-287.

(6) Hernandez-Ruiz, J.; Arnao, M. B.; Hiner, A. N. P.; GarciaCanovas, F.; Acosta, M. Catalase-like activity of horseradish peroxidase: relationship to enzyme inactivation by $\mathrm{H}_{2} \mathrm{O}_{2}$. Biochem. J. 2001, 354, 107-114.

(7) Bafana, A.; Dutt, S.; Kumar, S.; Ahuja, P. S. Superoxide dismutase: an industrial perspective. Crit. Rev. Biotechnol. 2011, 31, $65-76$.

(8) Zhu, X.; Niu, X. H.; Zhao, H. L.; Tang, J.; Lan, M. B. Immobilization of superoxide dismutase on Pt-Pd/MWCNTs hybrid modified electrode surface for superoxide anion detection. Biosens. Bioelectron. 2015, 67, 79-85.

(9) Pavlovic, M.; Rouster, P.; Szilagyi, I. Synthesis and formulation of functional bionanomaterials with superoxide dismutase activity. Nanoscale 2017, 9, 369-379.

(10) Rouster, P.; Pavlovic, M.; Szilagyi, I. Immobilization of Superoxide Dismutase on polyelectrolyte functionalized titania nanosheets. ChemBioChem 2018, 19, 404-410.

(11) Li, Z.; Wang, F.; Roy, S.; Sen, C. K.; Guan, J. J. Injectable, highly flexible, and thermosensitive hydrogels capable of delivering superoxide dismutase. Biomacromolecules 2009, 10, 3306-3316.

(12) Chen, Y. P.; Chen, C. T.; Hung, Y.; Chou, C. M.; Liu, T. P.; Liang, M. R.; Mou, C. Y. A new strategy for intracellular delivery of enzyme using mesoporous silica nanoparticles: Superoxide Dismutase. J. Am. Chem. Soc. 2013, 135, 1516-1523.

(13) Bobone, S.; Miele, E.; Cerroni, B.; Roversi, D.; Bocedi, A.; Nicolai, E.; Di Venere, A.; Placidi, E.; Ricci, G.; Rosato, N.; Stella, L. Liposome-Templated Hydrogel Nanoparticles as Vehicles for Enzyme-Based Therapies. Langmuir 2015, 31, 7572-7580.

(14) Singh, S.; Dubey, V. K. Multiwalled carbon nanotubesuperoxide dismutase conjugate towards alleviating induced oxidative stress. Int. J. Pept. Res. Ther. 2016, 22, 171-177.

(15) Zhang, Y.; Tang, A. D.; Yang, H. M.; Ouyang, J. Applications and interfaces of halloysite nanocomposites. Appl. Clay Sci. 2016, 119, $8-17$.

(16) Yuan, P.; Tan, D. Y.; Annabi-Bergaya, F. Properties and applications of halloysite nanotubes: recent research advances and future prospects. Appl. Clay Sci. 2015, 112, 75-93.

(17) Vergaro, V.; Abdullayev, E.; Lvov, Y. M.; Zeitoun, A.; Cingolani, R.; Rinaldi, R.; Leporatti, S. Cytocompatibility and uptake of halloysite clay nanotubes. Biomacromolecules 2010, 11, 820-826. 
(18) Santos, A. C.; Ferreira, C.; Veiga, F.; Ribeiro, A. J.; Panchal, A.; Lvov, Y.; Agarwal, A. Halloysite clay nanotubes for life sciences applications: From drug encapsulation to bioscaffold. Adv. Colloid Interface Sci. 2018, 257, 58-70.

(19) Lvov, Y.; Wang, W. C.; Zhang, L. Q.; Fakhrullin, R. Halloysite clay nanotubes for loading and sustained release of functional compounds. Adv. Mater. 2016, 28, 1227-1250.

(20) Rouster, P.; Dondelinger, M.; Galleni, M.; Nysten, B.; Jonas, A. M.; Glinel, K. Layer-by-layer assembly of enzyme-loaded halloysite nanotubes for the fabrication of highly active coatings. Colloids Surf., $B$ 2019, 178, 508-514.

(21) Chao, C.; Liu, J. D.; Wang, J. T.; Zhang, Y. W.; Zhang, B.; Zhang, Y. T.; Xiang, X.; Chen, R. F. Surface modification of halloysite nanotubes with dopamine for enzyme immobilization. ACS Appl. Mater. Interfaces 2013, 5, 10559-10564.

(22) Tully, J.; Yendluri, R.; Lvov, Y. Halloysite clay nanotubes for enzyme immobilization. Biomacromolecules 2016, 17, 615-621.

(23) Zhai, R.; Zhang, B.; Liu, L.; Xie, Y. D.; Zhang, H. Q.; Liu, J. D. Immobilization of enzyme biocatalyst on natural halloysite nanotubes. Catal. Commun. 2010, 12, 259-263.

(24) Shchukin, D. G.; Sukhorukov, G. B.; Price, R. R.; Lvov, Y. M. Halloysite nanotubes as biomimetic nanoreactors. Small 2005, 1, 510-513.

(25) Kumar-Krishnan, S.; Hernandez-Rangel, A.; Pal, U.; CeballosSanchez, O.; Flores-Ruiz, F. J.; Prokhorov, E.; de Fuentes, O. A.; Esparza, R.; Meyyappan, M. Surface functionalized halloysite nanotubes decorated with silver nanoparticles for enzyme immobilization and biosensing. J. Mater. Chem. B 2016, 4, 2553-2560.

(26) Hari, J.; Sarkozi, M.; Foldes, E.; Pukanszky, B. Long term stabilization of PE by the controlled release of a natural antioxidant from halloysite nanotubes. Polym. Degrad. Stab. 2018, 147, 229-236.

(27) Massaro, M.; Amorati, R.; Cavallaro, G.; Guernelli, S.; Lazzara, G.; Milioto, S.; Noto, R.; Poma, P.; Riela, S. Direct chemical grafted curcumin on halloysite nanotubes as dual-responsive prodrug for pharmacological applications. Colloids Surf., B 2016, 140, 505-513.

(28) Vergaro, V.; Lvov, Y. M.; Leporatti, S. Halloysite clay nanotubes for resveratrol delivery to cancer cells. Macromol. Biosci. 2012, 12, 1265-1271.

(29) Eskandarloo, H.; Arshadi, M.; Enayati, M.; Abbaspourrad, A. Highly efficient recovery of heparin using a green and low-cost quaternary ammonium functionalized halloysite nanotube. ACS Sustainable Chem. Eng. 2018, 6, 15349-15360.

(30) Tao, D.; Higaki, Y.; Ma, W.; Takahara, A. Halloysite nanotube/ polyelectrolyte hybrids as adsorbents for the quick removal of dyes from aqueous solution. Chem. Lett. 2015, 44, 1572-1574.

(31) Bahri-Laleh, N.; Sadjadi, S.; Poater, A. Pd immobilized on dendrimer decorated halloysite clay: Computational and experimental study on the effect of dendrimer generation, Pd valance and incorporation of terminal functionality on the catalytic activity. $J$. Colloid Interface Sci. 2018, 531, 421-432.

(32) Li, H.; Zhu, X. H.; Xu, J. F.; Peng, W.; Zhong, S. A.; Wang, Y. The combination of adsorption by functionalized halloysite nanotubes and encapsulation by polyelectrolyte coatings for sustained drug delivery. RSC Adv. 2016, 6, 54463-54470.

(33) Veerabadran, N. G.; Mongayt, D.; Torchilin, V.; Price, R. R.; Lvov, Y. M. Organized shells on clay nanotubes for controlled release of macromolecules. Macromol. Rapid Commun. 2009, 30, 99-103.

(34) Mitra, G. B.; Bhattacherjee, S. Structure of halloysite. Acta Crystallogr., Sect. B: Struct. Crystallogr. Cryst. Chem. 1975, 31, 28512857.

(35) Hassan, P. A.; Rana, S.; Verma, G. Making sense of Brownian motion: Colloid characterization by dynamic light scattering. Langmuir 2015, 31, 3-12.

(36) Zaccone, A.; Wu, H.; Lattuada, M.; Morbidelli, M. Correlation between colloidal stability and surfactant adsorption/association phenomena studied by light scattering. J. Phys. Chem. B 2008, 112, 1976-1986
(37) Kobayashi, M.; Skarba, M.; Galletto, P.; Cakara, D.; Borkovec, M. Effects of heat treatment on the aggregation and charging of Stöber-type silica. J. Colloid Interface Sci. 2005, 292, 139-147.

(38) Rouster, P.; Pavlovic, M.; Horvath, E.; Forro, L.; Dey, S. K.; Szilagyi, I. Influence of protamine functionalization on the colloidal stability of 1D and 2D titanium oxide nanostructures. Langmuir 2017, 33, 9750-9758.

(39) Bradford, M. M. Rapid and sensitive method for quantitation of microgram quantities of protein utilizing principle of protein-dye binding. Anal. Biochem. 1976, 72, 248-254.

(40) Beaucham, C.; Fridovich, I. Superoxide dismutase - improved assays and an assay applicable to acrylamide gels. Anal. Biochem. 1971, 44, 276-287.

(41) Byun, Y.; Chang, L. C.; Lee, L. M.; Han, I. S.; Singh, V. K.; Yang, V. C. Low molecular weight protamine: A potent but nontoxic antagonist to heparin/low molecular weight protamine. ASAIO J. 2000, 46, 435-439.

(42) Bauer, D.; Killmann, E.; Jaeger, W. Flocculation and stabilization of colloidal silica by the adsorption of poly-diallyldimethyl-ammoniumchloride (PDADMAC) and of copolymers of DADMAC with $\mathrm{N}$-methyl-N-vinyl acetamide (NMVA). Colloid Polym. Sci. 1998, 276, 698-708.

(43) Hyde, E. D. E.; Moreno-Atanasio, R.; Millner, P. A.; Neville, F. Surface charge control through the reversible adsorption of a biomimetic polymer on silica particles. J. Phys. Chem. B 2015, 119, 1726-1735.

(44) Hajdu, A.; Szekeres, M.; Toth, I. Y.; Bauer, R. A.; Mihaly, J.; Zupko, I.; Tombacz, E. Enhanced stability of polyacrylate-coated magnetite nanoparticles in biorelevant media. Colloids Surf., B 2012, 94, 242-249.

(45) Feng, L. L.; Stuart, M. C.; Adachi, Y. Dynamics of polyelectrolyte adsorption and colloidal flocculation upon mixing studied using mono-dispersed polystyrene latex particles. Adv. Colloid Interface Sci. 2015, 226, 101-114.

(46) Yi, P.; Pignatello, J. J.; Uchimiya, M.; White, J. C. Heteroaggregation of cerium oxide nanoparticles and nanoparticles of pyrolyzed biomass. Environ. Sci. Technol. 2015, 49, 13294-13303.

(47) Derjaguin, B.; Landau, L. D. Theory of the stability of strongly charged lyophobic sols nd of the adhesion of strongly charged particles in solutions of electrolytes. Acta Phys. Chim. 1941, 14, 633662.

(48) Bannister, J.; Bannister, W.; Wood, E. Bovine erythrocyte cupro-zinc protein 1. Isolation and general characterization. Eur. J. Biochem. 1971, 18, 178-186.

(49) Farmer, V. C.; Russel, J. D. The infra-red spectra of layer silicates. Spectroc. Acta 1964, 20, 1149-1173.

(50) Frost, R. L. Fourier-transform Raman-spectroscopy of kaolinite, dickite and halloysite. Clays Clay Miner. 1995, 43, 191-195.

(51) Mei, D. D.; Zhang, B.; Liu, R. C.; Zhang, Y. T.; Liu, J. D. Preparation of capric acid/halloysite nanotube composite as formstable phase change material for thermal energy storage. Sol. Energy Mater. Sol. Cells 2011, 95, 2772-2777.

(52) Wang, Y. Y.; Qiu, W. Y.; Wang, Z. B.; Ma, H. L.; Yan, J. K. Extraction and characterization of anti-oxidative polysaccharideprotein complexes from Corbicula fluminea through three-phase partitioning. RSC Adv. 2017, 7, 11067-11075.

(53) Szilagyi, I.; Berkesi, O.; Sipiczki, M.; Korecz, L.; Rockenbauer, A.; Palinko, I. Preparation, characterization and catalytic activities of immobilized enzyme mimics. Catal. Lett. 2009, 127, 239-247.

(54) Fakhrullina, G.; Khakimova, E.; Akhatova, F.; Lazzara, G.; Parisi, F.; Fakhrullin, R. Selective antimicrobial effects of curcumin@ halloysite nanoformulation: A caenorhabditis elegans study. ACS Appl. Mater. Interfaces 2019, 11, 23050-23064.

(55) Zhang, Y. M.; Li, Y. Q.; Zhang, Y. F.; Ding, D. Q.; Wang, L.; Liu, M.; Zhang, F. C. Thermal behavior and kinetic analysis of halloysite-stearic acid complex. J. Therm. Anal. Calorim. 2019, 135, $2429-2436$ 
(56) Sheldon, R. A.; van Pelt, S. Enzyme immobilisation in biocatalysis: why, what and how. Chem. Soc. Rev. 2013, 42, 62236235.

(57) Moura, F. A.; de Andrade, K. Q.; dos Santos, J. C. F.; Araujo, O. R. P.; Goulart, M. O. F. Antioxidant therapy for treatment of inflammatory bowel disease: Does it work? Redox Biol. 2015, 6, 617639.

(58) Moore, T. L.; Rodriguez-Lorenzo, L.; Hirsch, V.; Balog, S.; Urban, D.; Jud, C.; Rothen-Rutishauser, B.; Lattuada, M.; Petri-Fink, A. Nanoparticle colloidal stability in cell culture media and impact on cellular interactions. Chem. Soc. Rev. 2015, 44, 6287-6305.

(59) Vasti, C.; Bedoya, D. A.; Rojas, R.; Giacomelli, C. E. Effect of the protein corona on the colloidal stability and reactivity of LDHbased nanocarriers. J. Mater. Chem. B 2016, 4, 2008-2016. 